\title{
When they don't bite, we smell money: understanding malaria bednet misuse
}

\author{
KEITA HONJO ${ }^{1} \uparrow$, LUIS FERNANDO CHAVES ${ }^{1,2} * \dagger$, AKIKO SATAKE ${ }^{1}$, \\ AKIRA KANEKO ${ }^{3,4,5}$ and NOBORU MINAKAWA ${ }^{5}$ \\ ${ }^{1}$ Graduate School of Environmental Sciences, Hokkaido University, Sapporo 060-0810, Japan \\ ${ }^{2}$ Programa de Investigación en Enfermedades Tropicales, Escuela de Medicina Veterinaria, Universidad Nacional, \\ Heredia, Costa Rica \\ ${ }^{3}$ Island Malaria Group, Department of Microbiology, Tumor and Cell Biology, Karolinska Institutet, 17177 Stockholm, \\ Sweden \\ ${ }_{5}^{4}$ School of Medicine, Department of Medical Zoology, Osaka City University, Osaka 545-8585, Japan \\ ${ }^{5}$ Institute of Tropical Medicine (NEKKEN) Nagasaki University and Global COE Program, 1-12-4 Sakamoto, \\ Nagasaki 852-8523, Fapan
}

(Received 10 September 2012; revised 6 November 2012; accepted 7 November 2012; first published online 24 January 2013)

\section{S UMMARY}

Insecticide-treated nets (ITNs) are a major tool to control malaria. Over recent years increased ITN coverage has been associated with decreased malaria transmission. However, ITN 'misuse' has been increasingly reported and whether this emergent behaviour poses a threat to successful malaria control and elimination is an open question. Here, we use a game theory mathematical model to understand the possible roles of poverty and malaria infection protection by individual and emerging 'community effects' on the 'misuse' of malaria bednets. We compare model predictions with data from our studies in Lake Victoria Islands (LVI), Kenya and Aneityum, Vanuatu. Our model shows that alternative ITN use is likely to emerge in impoverished populations and could be exacerbated if ITNs become ineffective or when large 'community effects' emerge. Our model predicted patterns of ITN use similar to the observed in LVI, where 'misuse' is common and the high ITN use in Aneityum, more than 20 years after malaria elimination in 1990. We think that observed differences in ITN use may be shaped by different degrees of economic and social development, and educational components of the Aneityum elimination, where traditional cooperative attitudes were strengthened with the malaria elimination intervention and post-elimination surveillance.

Key words: Plasmodium, poverty, Pareto equilibrium, Nash equilibrium, insecticide treated nets, Kenya, Vanuatu.

\section{INTRODUCTION}

The Roll Back Malaria initiative (RBM) was launched in 1998 to tackle malaria, a disease with $3 \cdot 2$ billion people at risk of infection worldwide (World Health Organization, 2000). In 2000, African countries committed to providing proper treatment and insecticide-treated nets (ITNs, which are primarily bednets) to at least $60 \%$ of the highest malaria risk population by 2005, a goal raised to $80 \%$ by 2010 (RBM-Partnership, 2005). The mass ITN distribution campaign significantly reduced malaria-related morbidity and mortality (Lindblade et al. 2004; Fegan et al. 2007; O'Meara et al. 2008), and further scaling up ITN coverage is ongoing. However, some studies (Minakawa et al. 2008; Lover et al. 2011; O'Meara et al. 2011; Pulford et al. 2011) have reported ITN misuse as a potential explanation for partial success to increase net coverage, or the misuse of means, for example subsidized vouchers,

* Corresponding author: Graduate School of Environmental Sciences, Hokkaido University, Suite A701, Kita-10, Nishi-5, Kita-Ku, Sapporo, Hokkai-do, 0600810 Japan. Tel: +8111706 2267. Fax: +81 117064954 . E-mail: lchaves@ees.hokudai.ac.jp

$\uparrow$ These authors contributed equally to the manuscript. to obtain the ITNs (Tami et al. 2006). For instance, newly distributed LLINs are often patched together to make a large seine net (Fig. 1A), as old ones with holes are not effective for drying (Fig. 1B) and capturing fish (Fig. 1C) in fishing villages because of the net strength (Minakawa et al. 2008). Protecting plant crops (Fig. 1D) or granaries (Fig. 1E) with ITNs are becoming increasingly popular. Residents are now aware of the insecticidal and repellant effects of ITNs on crop pests. It is unclear how widely ITNs are used for other purposes, besides the plain misuse, e.g. as a sleeping mat (Fig. 1F), and a question remains as to whether this phenomenon hampers the ongoing efforts to reduce malaria transmission (Eisele et al. 2011).

Community effects may reduce transmission risk for people employing ITNs for purposes other than mosquito bite protection. For example, if some residents sleep under ITNs, in a proportion large enough to significantly decrease mosquito abundance (Howard et al. 2000; Hawley et al. 2003), that fraction of ITNs used for malaria prevention can shield all individuals in a community independently of the use given by an individual to his/her personal/household ITN(s). The income generated by alternative ITN use may further reduce the risk of malaria

Parasitology (2013), 140, 580-586. C Cambridge University Press 2013. The online version of this article is published within an Open Access environment subject to the conditions of the Creative Commons Attribution-NonCommercial-ShareAlike licence < http:// creativecommons.org/licenses/by-nc-sa/3.0/>. The written permission of Cambridge University Press must be obtained for commercial re-use. doi:10.1017/S0031182012002077 


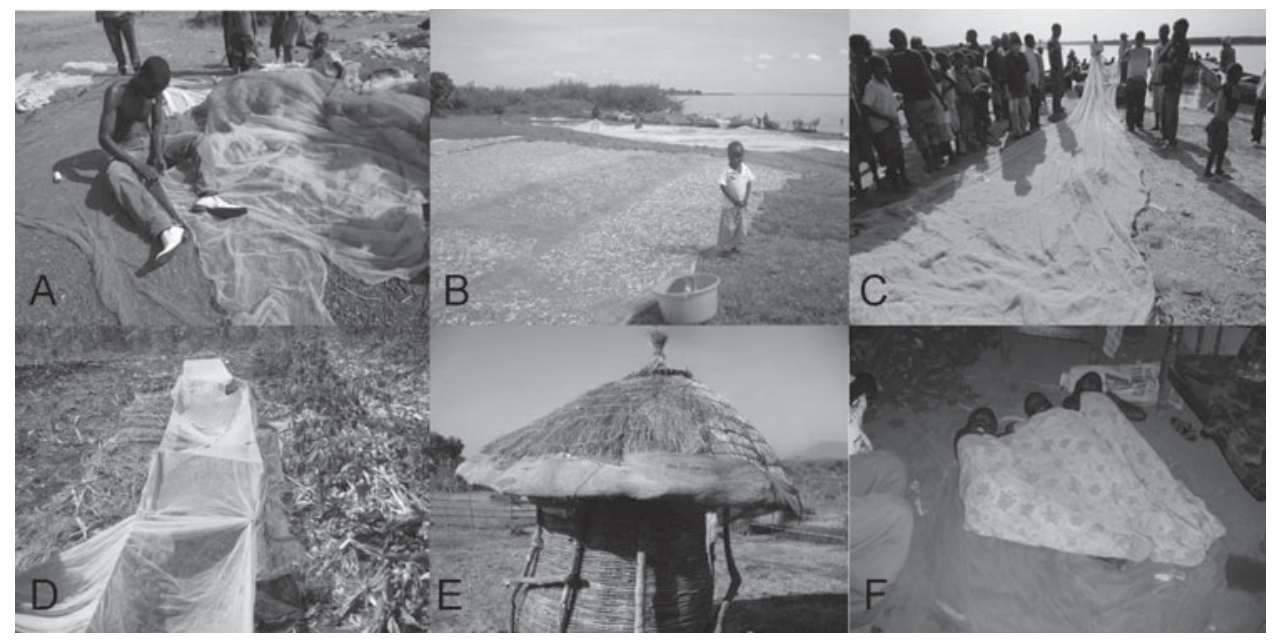

Fig. 1. Examples of alternative ITN uses. (A) Sewing bednets to create larger nets. (B) Drying fish. (C) Fishing. (D) Crop protection. (E) Granary protection. (F) Sleeping mat.

transmission or morbidity and mortality if the income is used for malaria and other infectious disease treatment, housing improvement and socioeconomic mobility, all factors associated with the reduction of malaria risk (Ijumba and Lindsay, 2001; Lindsay and Birley, 2004; Chaves and Koenraadt, 2010). Game theory, a conceptual framework widely used to understand behaviour in economics (Nash, 1950; Karlin, 1959) and ecological and evolutionary contexts (Smith and Price, 1973) offers modelling tools to understand the emergence of alternative ITN use by rendering an optimization between the benefits and risks of different ITN use. Game models allow the optimization of a player strategy reward through the derivation of Nash equilibria (Nash, 1950) and also the optimization of the public welfare by combining the strategies of all players in Pareto equilibria (Karlin, 1959). Here, we introduce a two-player game to understand the emergence of alternative ITN use. In the game, each of the two players uses or misuses its ITN for malaria prevention to optimize its own payoff, which we measure as an economic reward. In the model we assume that proper ITN use decreases malaria infection probability, while alternative ITN use increases labour productivity (e.g. income in US \$ per capita). We derive the Nash and Pareto equilibria to evaluate the individual and social impact of a player strategy. From the distribution of Pareto efficient Nash equilibria in the ITN use game, we found that alternative ITN use can optimize each player reward and public welfare simultaneously which we further illustrate with numerical solutions to our model and field data from Kenya and Vanuatu.

\section{THE MODEL}

In the next lines we define a 2-player (which can be also understood as a two strategy) ITN use game. First we define an expected payoff matrix for using/misusing an ITN, i.e. a mathematical set of formulae that are analysed to optimize the individual (Nash equilibrium) and collective (Pareto equilibrium) rewards. We then use the model to explore the economic rationality behind alternative ITN use. Through the model presentation we will use the term player to refer to the residents' use strategy in a malaria-endemic area with freely available ITNs.

\section{Data}

To test model predictions we used data on parasite rates (PR) and ITN use for malaria protection (ITNMP) from Aneityum, Vanuatu and Lake Victoria Islands (LVI), Kenya. For the analysis we used (PR) based on blood slide examination, which were about $1 / 3$ of the estimates with a rapid diagnostic test (RDT). Per cent ITNMP was based on ITN self-reported usage by residents, corrected by the percentage of the population covered with ITNs (see Table 1 for further details about coverage, use and PRs using BSE and RDT).

\section{Ethical approval}

This study was approved by the Vanuatu Department of Health, the Scientific Steering Committee and National Ethics Review Committee of the Kenya Medical Research Institute (SSC No. 1310 and 2131), and the ethics review committee of Nagasaki University.

\section{Expected payoff matrix}

The expected payoff matrix represents the set of strategies and rewards (commonly referred as payoffs in the game theory literature) that players can employ regarding a behaviour in a game model. In the ITN game, the 2 players have a common set of strategies denoted by $\mathrm{T}$ and $\mathrm{F}$, which correspond to ITN malaria protection use and misuse, respectively. Each 
Table 1. Insecticide-treated net (ITN) self-reported use, coverage and malaria parasite rates in Aneytium, Vanuatu and islands (Nghode, Takawiri, Kibougi, Mfangano) in Lake Victoria, Kenya

\begin{tabular}{|c|c|c|c|c|c|c|}
\hline Location & Year & Month & $\begin{array}{l}\text { Population } \\
\text { surveyed* }\end{array}$ & $\begin{array}{l}\text { Parasite } \\
\text { rate (per } 100 \\
\text { individuals) }\end{array}$ & $\begin{array}{l}\text { ITN } \\
\text { coverage }^{b}\end{array}$ & $\begin{array}{l}\text { ITN } \\
\text { use }^{c}\end{array}$ \\
\hline Aneityum ${ }^{19}$ & 1991 & January $^{\mathrm{d}}$ & 446 & 23 (NA) & 0 & na \\
\hline Aneityum $^{19}$ & 1991 & October/November ${ }^{\mathrm{e}}$ & 773 & $0 \cdot 004(\mathrm{NA})$ & 94 & 90 \\
\hline Aneityum & 2010 & July & 1123 & $0(0)$ & 100 & 97 \\
\hline Nghode & 2012 & February & 331 & $5(17)$ & 81 & 59 \\
\hline Takawiri & 2012 & February & 601 & $4(15)$ & 79 & 65 \\
\hline Kibougi & 2012 & February & 130 & $9(25)$ & 73 & 71 \\
\hline Mfangano & 2012 & February & 890 & $23(49)$ & 78 & 50 \\
\hline
\end{tabular}

* All surveys sampled representatively the demographic profile of the islands.

a The value outside the parentheses is the estimate based on blood slide examination, the value inside the parentheses is the estimate based on a Rapid Diagnostic test (Paracheck-Pf®) and na indicates not available.

b \% population owning a bednet.

c \% population using bednets independent of coverage.

d Pre-elimination baseline survey.

e Post-elimination baseline survey.

player chooses a strategy ( $\mathrm{T}$ or $\mathrm{F}$ ) based on his/her malaria infection risk, labour productivity, and expected payoff. Thus, the ITN game can have 4 profiles, which result from the combination of the strategies by the 2 players: $(\mathrm{T}, \mathrm{T}),(\mathrm{T}, \mathrm{F}),(\mathrm{F}, \mathrm{T})$ and $(\mathrm{F}, \mathrm{F})$.

The relation between the ITN game profiles and the malaria infection risk can be represented by an infection probability (IP) matrix (Fig. 2A). In this matrix we define an IP $P$, which can take any value between 0 and 1 (i.e. $1 \geqslant P \geqslant 0$ ). $P$ can be interpreted as the probability of malaria infection by an individual in the setting where she/he resides. To make the connection with epidemiological literature, $P$ could be seen as a function of malaria infection risk factors, i.e. the higher the odds of an individual being infected, the higher the value of $P$. The use of an ITN by a player is assumed to reduce the individual probability of infection by $\alpha_{1}$, as observed in numerous studies (Howard et al. 2000; Hawley et al. 2003; Lindblade et al. 2004; Fegan et al. 2007), and the use of ITNs by other residents in the community can lead to an emergent 'community effect' (Howard et al. 2000; Kaneko et al. 2000; Hawley et al. 2003; Fegan et al. 2007; Chaves et al. 2008) that further reduces $P$ by a factor $\left(\alpha_{2}\right)^{n}$, where $n$ is the number of players that use the ITN for malaria prevention. Thus, the 'community effect' is null when no players use bednets and the magnitude of its impact increases as more individuals use the ITNs for malaria prevention. The $\alpha$ parameters can take any value above 0 and below 1 (i.e. $0<\alpha_{i}<1, i=1,2$ ). We can then define a labour productivity matrix (Fig. 2 B) that quantifies the utility of labour in malaria uninfected players, $L$, and the increased utility $\beta$ by using bednets for alternative purposes to malaria prevention. Finally, with these two matrices we can define the expected payoff matrix (Fig. 2C) as the
(A)

\begin{tabular}{|c|c|c|}
\hline Player 1 & $\mathrm{T}$ & $\mathrm{F}$ \\
\hline $\mathrm{T}$ & $\alpha_{1} \alpha_{2}^{2} P, \alpha_{1} \alpha_{2}^{2} P$ & $\alpha_{1} \alpha_{2} P, \alpha_{2} P$ \\
\hline $\mathrm{F}$ & $\alpha_{2} P, \alpha_{1} \alpha_{2} P$ & $P, P$ \\
\hline
\end{tabular}

(B)

\begin{tabular}{|c|c|c|}
\hline Player 1 & $\mathrm{T}$ & $\mathrm{F}$ \\
\hline $\mathrm{T}$ & $L, L$ & $L, \beta L$ \\
\hline $\mathrm{F}$ & $\beta L, L$ & $\beta L, \beta L$ \\
\hline
\end{tabular}

(C)

\begin{tabular}{|c|c|c|}
\hline Player 1 Player 2 & $\mathrm{T}$ & $\mathrm{F}$ \\
\hline $\mathrm{T}$ & $\begin{array}{c}\left(1-\alpha_{1} \alpha_{2}^{2} P\right) L, \\
\left(1-\alpha_{1} \alpha_{2}^{2} P\right) L\end{array}$ & $\begin{array}{c}\left(1-\alpha_{1} \alpha_{2} P\right) L \\
\left(1-\alpha_{2} P\right) \beta L\end{array}$ \\
\hline $\mathrm{F}$ & $\begin{array}{c}\left(1-\alpha_{2} P\right) \beta L \\
\left(1-\alpha_{1} \alpha_{2} P\right) L\end{array}$ & $\begin{array}{c}(1-P) \beta L \\
(1-P) \beta L\end{array}$ \\
\hline
\end{tabular}

Fig. 2. Deriving an expected payoff matrix for the ITN use game. (A) Infection probability (IP) matrix. $\mathrm{P}$ is the malaria infection probability of the players in the absence of ITNs. The parameters $\alpha_{1}$ and $\alpha_{2}$ denote the individual and community effects of ITN use for malaria protection, respectively. To read this and the subsequent matrices the strategy of player 1 is presented in the rows, and of player 2 in the columns. The matrix value for player 1 is the first entry in a given cell. (B) Labour productivity matrix. $L$ is the labour productivity (which can be measured in US $\$$ per capita) of the players without an ITN, $L$ can take any positive value (i.e. $L>0$ ). The parameter $\beta$ denotes the $\beta$-fold increment of $L$ when a player gives an alternative use to his/her ITN, $\beta$ is assumed to be larger than 1 (i.e. $\beta>1$ ). (C) Expected payoff matrix. This matrix is the Hadamard product (i.e. matrix-element-wise product) of the complement of the IP matrix (i.e. 1 - IP matrix) and the Labour productivity matrix for each player. 
product of the probability of not being infected with malaria (1-probability of malaria infection) and the labour utility. The model assumes a perfect knowledge of the costs and benefits for different ITN uses. The 2-player model has the advantage of rendering general results independently of whether the impacts of ITN use for malaria protection are density or frequency dependent, since results for small $n$ are independent of density/frequency-dependent pathogen transmission (Antonovics et al. 1995) and, in general, 2-player games are useful tools to understand the emergence of different behaviours in a population (Smith and Price, 1973).

\section{Distribution of Nash equilibria}

Nash equilibria are the profiles (combination of player strategies) from which any player has no incentive to deviate, because his/her payoff is maximized in response to the other player strategy (Nash, 1950; Smith and Price, 1973). Our model has 3 Nash equilibria that were derived by establishing the conditions when given a profile, none of the players can increase his/her payoff by changing his/her strategy. For example, in the case of the profile ( T, T), i.e. when both players use their ITNs for malaria prevention we have that $(\mathrm{T}, \mathrm{T})$ is a Nash equilibrium when the following inequality holds:

$\left(1-\alpha_{1}\left(\alpha_{2}\right)^{2} P\right) L>\left(1-\alpha_{2} P\right) \beta L$.

This implies a higher payoff for any player if he/she uses the ITN for malaria prevention than if he/she chooses to profit from an alternative ITN use. Here it is worth highlighting that payoffs are independent of labour productivity $(L)$, which cancels out on both sides of Equation (1). Nevertheless, payoffs are proportional to the increase $(\beta)$ of $L$ by the alternative ITN use. From expression (1) a threshold for IP $\left(P_{R}\right)$ can be derived which ensures that both players will use their ITNs for malaria prevention when $P>P_{R}$ :

$P_{R}=\frac{\beta-1}{\alpha_{2}\left(\beta-\alpha_{1} \alpha_{2}\right)}$.

Following a similar procedure, $P_{L}$, an IP threshold where $(\mathrm{F}, \mathrm{F})$, both players giving alternative uses to their ITNs, is a Nash Equilibrium when $P<P_{L}$, can be derived:

$P_{L}=\alpha_{2} P_{R}$.

Finally, the profiles $(\mathrm{T}, \mathrm{F})$ and $(\mathrm{F}, \mathrm{T})$ are Nash equilibria when $P$ follows the following condition:

$P_{L} \leqslant P \leqslant P_{R}$.

Equation (4) implies the emergence of 'free rider' Nash equilibria, where a player with the strategy $F$ benefits from the alternative use of his/her ITN and from the 'community effect' in malaria protection that emerges by the use of ITNs for malaria prevention by a player with the strategy $\mathrm{T}$.

\section{Distribution of Pareto equilibria}

A Pareto equilibrium is a combination of player payoffs that is efficient for the public welfare (Karlin, 1959), in the context of this study meaning it conduces to a reduction of malaria infection risk in a community. To find the Pareto equilibria we solved several inequalities comparing the different profiles of the ITN game (see Supplementary material, online version only, for a detailed and mathematically rigorous derivation). Our analysis showed the Nash equilibria to be Pareto efficient with the exception of the equilibria for the profile $(\mathrm{F}, \mathrm{F})$, where a region with a social dilemma (SD), i.e. where the whole community benefits by a player changing his/her strategy, emerges when:

$P_{L}^{*}=\frac{\beta-1}{\beta-\alpha_{1} \alpha_{2}^{2}}$.

By definition, $P_{L}^{*}<P_{L}$, is the difference between these two thresholds defining the range of malaria infection probability over which a SD emerges, and is expected to be wider as the number of players increases in a community (Nash, 1950).

\section{Model implications}

Our model can be used to illustrate the influence of many factors that may underpin patterns of ITN misuse. Malaria is a disease entrenched among the poorest nations in the globe (Chaves and Koenraadt, 2010) and the alternative use of ITNs could represent a significant increase in a household income. Figure 3A illustrates how the range of malaria infection probability $(P)$ where all players prefer to use ITNs for purposes other than malaria protection doubles its width when a $20 \%$ increase in the player income is derived by an alternative ITN use, a figure that may be realistic for the poorest of the poor in many developing nations. This is the case even when assuming that ITN use reduces by $40 \%$ (i.e. $\alpha_{1}=0.60$, e.g. see Killeen et al. (2007)) the probability of malaria infection, a value within the range of observed outcomes for ITN trials (Lindblade et al. 2004). The free-rider behaviour is expected to become increasingly common as the 'community effect' increases (Fig. 3B and C), in an exacerbated manner as the protection level by individual ITN use diminishes (i.e. $\alpha_{1}$ increasing towards 1, Fig. 3C).

\section{$I T N$ use and parasite rates (PR) in Vanuatu and Kenya islands, do they follow the model?}

Table 1 shows data from malaria surveys made before (PRE) and after (POST) the 1991 elimination intervention, and 2010, in Aneityum (a Vanuatu island) and from 2012 in several LVI (Ngodhe, Takawiri, Kibuogi, Mfangano). In Aneityum, before 
(A)

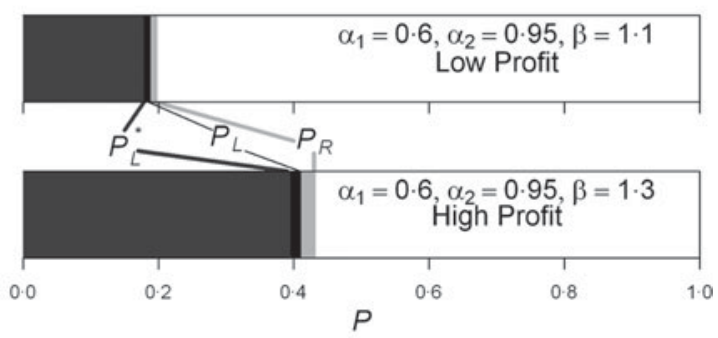

(B)

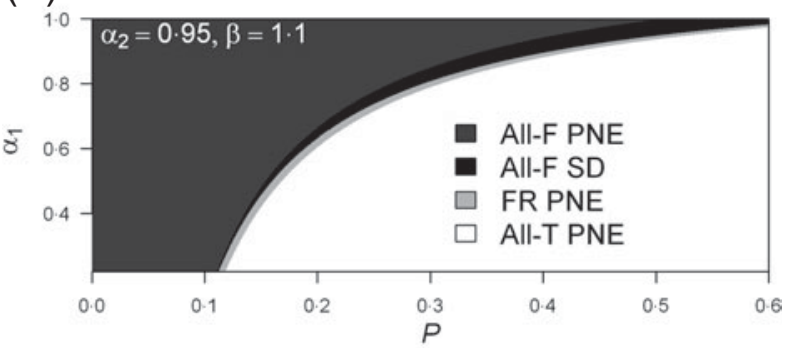

(C)

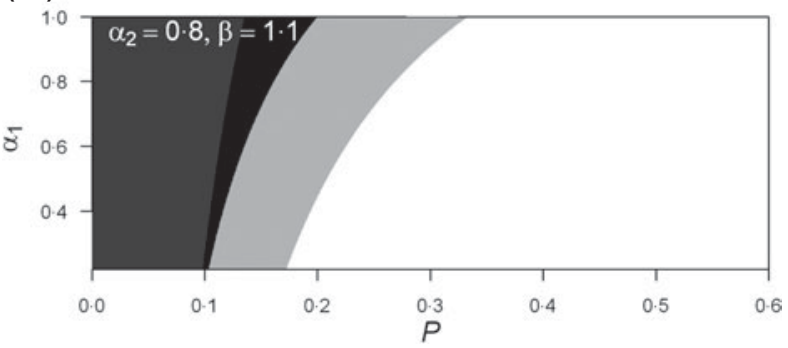

Fig. 3. Pareto efficient Nash equilibria (PNE) and Social Dilemma (SD). (A) Equilibria as function of the infection probability, $P$, the top panel illustrates a case where profitability for alternative ITN use is low (income increases by $10 \%$, i.e. $\beta=1 \cdot 1$ ), the bottom panel represents a case of higher profitability for the alternative bednet use (income increases by $30 \%$, i.e. $\beta=1 \cdot 3$ ). In the two panels the thresholds $P_{R}, P_{L}$ and $P_{L}^{*}$ are indicated (see the main text for an explanation of the thresholds). The legend in panel B applies to panels A, B and C: All-T (All-F) are the equilibria where all players (do not) use the ITN for malaria protection, FR are the free-rider equilibria. (B) Equilibria as function of $P$ and individual bednet protection $\left(\alpha_{1}\right)$ in a setting with a low level of additional protection via a 'community effect' ( $5 \%$ i.e. $\left.\alpha_{2}=0.95\right)$. (C) Equilibria as function of $P$ and individual bednet protection $\left(\alpha_{1}\right)$ in a setting with a high level of additional malaria protection via a community effect $(20 \%$, i.e. $\left.\alpha_{2}=0 \cdot 80\right)$.

the 1991 elimination trial, PR was slightly above 20\% and ITN coverage was null (Table 1). As part of the elimination trial coverage was raised to $94 \%$ and use was around 90\% (Kaneko et al. 2000), and currently coverage is $100 \%$ with $97 \%$ use. In Vanuatu, current levels of use are in accordance with equation (2) when the parameter $\beta \rightarrow 1$, i.e. when there is no significant increase of labour productivity by an alternative ITN use and with a transmission close to 0 all players will use the ITN for malaria protection (i.e. $P_{R} \rightarrow 0$ ). Nevertheless, our model fails to explain the patterns observed in Vanuatu under the assumption of $\beta>1$. The situation in LVI reflects a higher coverage and use of ITNs than that observed in Aneityum prior to the elimination trials (Table 1), yet misuse may be higher, since around $20 \%$ of the ITNs are not being used for malaria protection (Table 1), and only around $50 \%$ of the population use ITNs for malaria protection. ITN use patterns in LVI resemble our model predictions when $\beta>1$.

\section{ISCUSSION}

Our model clearly indicates that ITN use for malaria protection can be thwarted in settings of extreme poverty, where an increase in labour productivity by an alternative ITN use can offset the perceived benefits of avoiding malaria infection. The model also shows that alternative ITN uses are expected to emerge as coverage and concomitant 'community effects' become more common, or if ITNs become unprotective, for example, by the emergence of insecticide-resistant mosquitoes (Kawada et al. 2011). We also showed that alternative ITN use is not necessarily detrimental for an endemic community, especially for low and moderate levels of malaria infection risk, since those strategies are Pareto efficient, in the context of this study meaning that alternative ITN use is not detrimental for the community as a whole. Our model also shows that as malaria risk further decreases, social dilemmas, i.e. situations where individual behaviours can improve a situation for a community as a whole, are likely to emerge, especially when the use of ITNs could be crucial to render elimination feasible (Smith et al. 2009), because they are not optimal from the perspective of non-cooperative individual residents. However, the only data we have available for a current low malaria risk area, formerly hyperendemic, i.e. Aneityum island in Vanuatu (Kaneko et al. 2000), suggest that the non-cooperative behaviour assumed in our model is not likely to interfere with ITN use for malaria protection when there is a likely small proportional increase of labour productivity by alternative ITN use. In Aneityum ITN use is very high $(>95 \%)$ well after elimination, probably because of the educational component of the elimination trial aimed at strengthening cooperative practices and promoting community participation of residents during the trial and subsequent malaria freedom (Kaneko, 2010). Also, Vanuatu has a moderate level of human development, where economic development is more sustainable, socially equitable and conducive to a higher standard of living (e.g. better education and access to services) than in most sub-Saharan African nations (UNDP, 2011), which makes unrealistic the scenario of significant increases to individual labour productivity by using an ITN for a purpose other than malaria protection. Thus, high ITN use for malaria protection could also reflect 
the relative higher well-being of the Vanuatu population when compared with most nations in subSaharan Africa. In fact, although coverage before the 1991 elimination trial of Vanuatu $(0 \%)$ was far from being as high as it is currently in LVI $(>70 \%)$, current ITN use is well over $(>97 \%)$ what we can observe in LVI $(>50 \%)$, which indicates a proportionally larger ITN misuse in LVI than that which we have observed in Vanuatu over the years. Nevertheless, issues of perceived mosquito annoyance as triggers of ITN use, a topic not explored by our current model, need to be further explored.

Regarding the robustness of our results (Levins, $1968,2006)$, i.e. whether our inferences remain the same under different or more elaborated assumptions, we can affirm that our major result, that ITN alternative use is a rational behaviour in impoverished settings, holds when the game is explicitly extended to $n$ players. However, some quantitative differences can be expected in thresholds for social dilemmas and other Pareto equilibria that become a direct function of the $n$ players. Nonetheless, effects of $n$ on ITN use are beyond our research goals in this contribution and will be presented elsewhere.

Finally, results from our model, in addition to common observations on ITN use, where 'misuse' is commonly related to alternative uses aimed at increasing labour productivity (Minakawa et al. 2008; Lover et al. 2011; Pulford et al. 2011), make us believe that malaria elimination efforts will be more likely to achieve success if interventions are embedded within a larger effort aimed at improving the well-being of endemic populations (Chaves and Koenraadt, 2010), since they can improve the adherence to interventions and have indirect effects, such as better access to improved healthcare (Ijumba and Lindsay, 2001), housing and others (Chaves and Koenraadt, 2010), that can further increase the odds of successful malaria control or elimination.

\section{ACKNOWLEDGEMENTS}

We thank Professor Toshihiko Sunahara for summarizing the results from the 2010 Aneityum malaria survey, Ms Sayaka Shimada for summarizing the results of the 2012 LVI malaria survey, Dr François Feugier and Professor Takenori Takada for valuable comments about game theory and $\mathrm{Mr}$ Gabriel Dida for his valuable comments and insights on ITN use.

\section{FINANCIAL SUPPORT}

This project was supported by Nagasaki University. K.H. and L.F.C. are supported by fellowships from the Japan Society for the Promotion of Science. All authors declare no competing interests.

\section{REFERENCES}

Antonovics, J., Iwasa, Y. and Hassell, M. P. (1995). A generalized model of parasitoid, venereal, and vector-based transmission processes. American Naturalist 145, 661-675.
Chaves, L. F., Kaneko, A., Taleo, G., Pascual, M. and Wilson, M. L. (2008). Malaria transmission pattern resilience to climatic variability is mediated by insecticide-treated nets. Malaria Fournal 7, 100 .

Chaves, L.F. and Koenraadt, C. J. M. (2010). Climate change and highland malaria: fresh air for a hot debate. Quarterly Review of Biology 85, 27-55.

Eisele, T. P., Thwing, J. and Keating, J. (2011). Claims about the misuse of insecticide-treated mosquito nets: are these evidence-based? PLoS Med $\mathbf{8}$, e1001019.

Fegan, G. W., Noor, A.M., Akhwale, W.S., Cousens, S. and Snow, R. W. (2007). Effect of expanded insecticide-treated bednet coverage on child survival in rural Kenya: a longitudinal study. Lancet 370, 1035-1039.

Hawley, W. A., Phillips-Howard, P.A., ter Kuile, F. O., Terlouw, D. J., Vulule, J.M., Ombok, M., Nahlen, B. L., Gimnig, J. E., Kariuki, S. K., Kolczak, M.S. and Hightower, A. W. (2003). Community-wide effects of permethrin-treated bed nets on child mortality and malaria morbidity in western Kenya. American Fournal of Tropical Medicine and Hygiene 68, 121-127.

Howard, S. C., Omumbo, J., Nevill, C., Some, E. S., Donnelly, C. A. and Snow, R.W. (2000). Evidence for a mass community effect of insecticide-treated bednets on the incidence of malaria on the Kenyan coast. Transactions of the Royal Society of Tropical Medicine and Hygiene 94, $357-360$.

Ijumba, J. N. and Lindsay, S. W. (2001). Impact of irrigation on malaria in Africa: paddies paradox. Medical and Veterinary Entomology $15,1-11$.

Kaneko, A. (2010). A community-directed strategy for sustainable malaria elimination on islands: short-term MDA integrated with ITNs and robust surveillance. Acta Tropica 114, 177-183.

Kaneko, A., Taleo, G., Kalkoa, M., Yamar, S., Kobayakawa, T. and Björkman, A. (2000). Malaria eradication on islands. Lancet 356, 1560-1564.

Karlin, S. (1959). Mathematical Methods and Theory in Games, Programming, and Economics. Volume I: Matrix Games, Programming, and Mathematical Economics. Addison-Wesley Pub. Co., Reading, MA, USA.

Kawada, H., Dida, G. O., Ohashi, K., Komagata, O., Kasai, S., Tomita, T., Sonye, G., Maekawa, Y., Mwatele, C., Njenga, S. M., Mwandawiro, C., Minakawa, N. and Takagi, M. (2011). Multimodal pyrethroid resistance in malaria vectors, Anopheles gambiae s.s., Anopheles arabiensis, and Anopheles funestus s.s. in Western Kenya. PLoS ONE 6, e22574.

Killeen, G. F., Smith, T.A., Ferguson, H.M., Mshinda, H., Abdulla, S., Lengeler, C. and Kachur, S. P. (2007). Preventing childhood malaria in Africa by protecting adults from mosquitoes with insecticide-treated nets. PLoS Med 4, e229.

Levins, R. (1968). Evolution in Changing Environments. Some Theoretical Explorations. Princeton University Press, Princeton, NJ, USA.

Levins, R. (2006). Strategies of abstraction. Biology $\odot$ Philosophy 21, 741-755.

Lindblade, K. A., Eisele, T.P., Gimnig, J. E., Alaii, J. A., Odhiambo, F., ter Kuile, F. O., Hawley, W. A., Wannemuehler, K. A., Phillips-Howard, P. A., Rosen, D. H., Nahlen, B. L., Terlouw, D. J., Adazu, K., Vulule, J.M. and Slutsker, L. (2004). Sustainability of reductions in malaria transmission and infant mortality in Western Kenya with use of insecticidetreated bednets. Fournal of the American Medical Association 291, 25712580 .

Lindsay, S. and Birley, M. (2004). Rural development and malaria control in sub-Saharan Africa. EcoHealth 1, 129-137.

Lover, A., Sutton, B., Asy, A. and Wilder-Smith, A. (2011). An exploratory study of treated-bed nets in Timor-Leste: patterns of intended and alternative usage. Malaria fournal 10, 199.

Minakawa, N., Dida, G., Sonye, G., Futami, K. and Kaneko, S. (2008). Unforeseen misuses of bed nets in fishing villages along Lake Victoria. Malaria Fournal 7, 165.

Nash, J. F. (1950). Equilibrium points in n-person games. Proceedings of the National Academy of Sciences, USA 36, 48-49.

O'Meara, W. P., Bejon, P., Mwangi, T. W., Okiro, E. A., Peshu, N., Snow, R. W., Newton, C. R. J. C. and Marsh, K. (2008). Effect of a fall in malaria transmission on morbidity and mortality in Kilifi, Kenya. Lancet 372, 1555-1562.

O'Meara, W. P., Smith, N., Ekal, E., Cole, D. and Ndege, S. (2011). Spatial distribution of bednet coverage under routine distribution through the Public Health Sector in a Rural District in Kenya. PLoS ONE 6, e25949. 
Pulford, J., Hetzel, M., Bryant, M., Siba, P. and Mueller, I. (2011). Reported reasons for not using a mosquito net when one is available: a review of the published literature. Malaria fournal 10, 83 .

RBM-Partnership (2005). Roll Back Malaria Global Strategic Plan 2005-2015. RBM Partnership Secretariat, Geneva, Switzerland.

Smith, D. L., Hay, S. I., Noor, A. M. and Snow, R. W. (2009). Predicting changing malaria risk after expanded insecticide-treated net coverage in Africa. Trends in Parasitology 25, 511-516.

Smith, J. M. and Price, G. R. (1973). The logic of animal conflict. Nature, London 246, 15-18.
Tami, A., Mbati, J., Nathan, R., Mponda, H., Lengeler, C. and Armstrong Schellenberg, J.R. (2006). Use and misuse of a discount voucher scheme as a subsidy for insecticide-treated nets for malaria control in southern Tanzania. Health Policy and Planning 21, $1-9$.

UNDP (2011). Human Development Report 2011 Sustainability and Equity: A Better Future for All. United Nations Development Programme, New York, USA.

World Health Organization (2000). The African Summit on Roll Back Malaria. World Health Organization, Geneva, Switzerland. 